\title{
Transatlantica
}

Revue d'études américaines. American Studies Journal

\section{Liliane Louvel et Catherine Rannoux (éds). La} Réticence.

Poitiers : Presses Universitaires de Rennes, « La Licorne », 2004. 404 p. 22 Euros.

\section{Sophie Chiari-Lasserre}

\section{(2) OpenEdition}

Journals

\section{Édition électronique}

URL : http://journals.openedition.org/transatlantica/858

DOI : $10.4000 /$ transatlantica.858

ISSN : $1765-2766$

Éditeur

AFEA

\section{Référence électronique}

Sophie Chiari-Lasserre, «Liliane Louvel et Catherine Rannoux (éds). La Réticence. », Transatlantica [En ligne], 1 | 2005, mis en ligne le 22 avril 2006, consulté le 29 avril 2021. URL : http://

journals.openedition.org/transatlantica/858; DOI : https://doi.org/10.4000/transatlantica.858

Ce document a été généré automatiquement le 29 avril 2021.

\section{(c) (i) $\odot$}

Transatlantica - Revue d'études américaines est mis à disposition selon les termes de la licence Creative Commons Attribution - Pas d'Utilisation Commerciale - Pas de Modification 4.0 International. 


\title{
Liliane Louvel et Catherine Rannoux (éds). La Réticence.
}

Poitiers : Presses Universitaires de Rennes, «La Licorne », 2004. 404 p. 22 Euros.

\author{
Sophie Chiari-Lasserre
}

1 Les vingt-quatre études réunies et présentées par Liliane Louvel et Catherine Rannoux dans le numéro 68 de $L a$ Licorne sont très hétéroclites, et sondent tous les genres et les « sous-genres » de la littérature et de l'art. Mais toutes aussi variées soient-elles, elles explorent avec une grande rigueur scientifique une figure paradoxale, où se conjuguent non seulement le manque, le silence et l'interruption, mais aussi les "tout dire", l'épuisement du sens et la prolifération. C'est donc sans parti-pris, en termes de dynamique et de dialectique, que va s'ébaucher dans les pages de l'ouvrage une réflexion tout en nuances sur la réticence. Voici donc vingt-quatre définitions d'une figure qui se dérobe à la lecture, mais qui nous fascine précisément parce qu'elle demeure insaisissable.

2 La première partie du volume fait la part belle aux linguistes, privilégiant la philosophie du langage. Elle débute par un article éclairant dans lequel Jean-Jacques Lecercle chasse les idées reçues, qui ont généralement tendance à faire du langage un simple instrument de communication. Il cherche alors à promouvoir une autre philosophie, en explorant les liens complexes entre silence et réticence, puis en faisant une taxinomie de la réticence où sont abordés le vague, l'euphémisme, ou le tabou. Par le biais d'exemples extrêmement variés (tirés de Montaigne, des Monthy Python, de Vita Sckville West ou de Jane Austen) l'auteur propose enfin des exemples flagrants de réticence, où le jeu social prend toute son importance. Ronald Shusterman se pose quant à lui une autre question: la réticence peut-elle, ou doit-elle, prévaloir sur l'expression? En ce qui concerne les arts plastiques, pour tout artiste, le fait de taire quelque chose est impossible à partir du moment où une œuvre existe. Le cas de la littérature semble plus compliqué puisque beaucoup confondent encore l'œuvre réticente, et celle mettant en scène la réticence. La première est un échec; la seconde en revanche relève toujours du mode du dire. Cette habile démonstration s'enchaîne 
avec un article signé par Sabine Pétillon qui, à partir de quelques manuscrits de Roland Barthes, étudie les mécanismes de la réticence, en prêtant une attention particulière aux modes d'inscriptions de la rature. Sont par exemple envisagés les différents types d'opération scripturaires permis par la rature, illustrations à l'appui. La communication de Simone Rinzler explore d'autres pistes: les énoncés dans lesquels l'aspect passif peut être perçu comme une figure de la réticence. L'auteur analyse deux tendances : « la réticence des locuteurs à entrer ou à rester dans le pacte dialogique » et les multiples "réticences à dire le monde » (53). Elle se penche sur les verbalisations difficiles, révélant des formes de réticences sexuelles, religieuses, ou même feintes dans le but de manipuler autrui. Le bilan de cette analyse est très clair: "le recours au passif, par rapport au choix du silence absolu signale que l'énonciateur souhaite maintenir le pacte dialogique » (70).

La deuxième partie du volume change d'orientation, et axe ses articles sur les "Origines Antiques ». Michel Briand se penche sur la littérature grecque ancienne à partir d'un roman grec sophistique, Le Roman de Leucippé et Clitophon d'Achille Tatius. On apprend que l'idéal philosophique, dans la littérature classique, est fréquemment représenté par un personnage réticent, qui forme un couple d'opposés avec le personnage fanfaron. La réticence reste une vertu que seuls possèdent les héros, mais on apprend comment cette notion peut également devenir sujette à parodie. L'intervenante suivante, Emmanuelle Valette-Cagnac, relit les traités de Cicéron. Ce faisant, elle constate que lorsque le discours s'interrompt volontairement, l'auditoire réagit face au vide, et le comble en puisant dans son imagination. La réticence est par conséquent l'atout des orateurs charismatiques, et elle permet aussi de limiter le discours afin que les auditeurs restent concentrés. L'auteur effectue donc ici avec succès « une sorte d'anthropologie de la réticence » (120) qui met bien en valeur la dimension pragmatique de l'oratio romaine. La contribution suivante s'interroge sur la réticence au cœur de la maïeutique socratique. A partir des textes de Platon, Christophe Laudou émet en effet une hypothèse séduisante : la dialectique confèrerait une place de choix à la coupure, afin de nous faire accéder au vrai. L'auteur utilise d'abord la métaphore de la faiseuse d'ange pour montrer que le dialogue, même s'il est achevé, reste une naissance. Or, toute naissance nécessite de "couper le cordon " (127). L'interruption du discours, à un moment donné, est donc une nécessité, mais c'est toujours à l'autre qu'il revient d'interrompre la parole de celui qui s'exprime. Georges Kliebenstein s'attaque quant à lui à " la métaphore quantitative du mi-dire " (136) afin de voir en quoi consiste l'hémisémie. A mi-chemin entre le " presque rien » et le "déjà trop » (139), la réticence aurait partie liée avec la nature érotique du langage. Klibenstein se propose d'établir ensuite des catégories taxinomiques de la réticence (absolue, provisoire, complétée par son destinateur ou par le destinataire) afin de rendre compte de fonctionnements spécifiques. En fin de compte, la réticence, en réalité, n'existerait pas, et cela pour deux raisons opposées. Tout d'abord, un texte ne peut jamais tout dire (la littérature ne serait alors que réticences continuelles). Et paradoxalement, c'est lorsqu'il dit les choses à moitié que, précisément, il dit tout...

4 Liliane Louvel et Catherine Rannoux nous proposent de continuer par les « Modernités Poétiques ", partie commencée par l'étude d'un sonnet anti-bonapartiste de Rimbaud. Alors que le poète s'oppose à l'image de la Nature façonnée par les Romantiques, JeanPierre Bobillot examine la réticence du locuteur dans le poème. Il se penche ensuite sur les Réparties de la Nina, en y examinant les cas d'interruption, avant d'enchaîner sur les 60 Respirations et Brèves rencontres de Bernard Heidsieck, texte truffé de 
ressaisissements, de coupures et de re-formulations visant à découvrir les béances de la langue (qu'elle soit ordinaire ou littéraire). De façon très convaincante, Colette Camelin, avec "le dénudement de la parole dans Sol Absolu de Lorand Gaspard", explique comment, à l'opposé du discours dogmatique toujours dense, le silence permet le déploiement de la respiration, et respecte le mouvement des choses. La poésie est par nature réticente au développement, et c'est la quête de la simplicité qui transparaît à travers le silence : quelques mots suffisent pour faire jaillir l'émotion. Le dernier article de cette partie est signé Michel Favriaud : il envisage d'abord la configuration en creux de la réticence dans Le Bol du pèlerin, texte où Jacottet fait siennes deux vertus essentielles, la patience et le risque. Il montre ensuite comment l'objet, le sujet et le discours sont affectés par la réticence dans ce poème empreint de modalisations et de gradations, avant de développer autre forme de réticence, englobant la négation.

Un autre volet esthétique laisse ensuite place à l'image et à la scène ; c'est Geneviève Joly qui ouvre le bal avec une étude centrée sur une pièce du Norvégien Jon Fosse, Quelqu'un va venir. S'érigeant contre l'interprétation de la réticence au théâtre comme une forme de minimalisme, elle propose une lecture de cette figure relevant du mode du dire. Dans le drame de Fosse, la parole est certes tissée de béances, d'ambiguïtés, de redondances et de re-formulations. Tout, de la mise en page, du jeu scénique, jusqu'à la scénographie de cette pièce, laisse surgir les non-dits du texte. L'auteur convient toutefois que le spectateur, constamment mobilisé, peut être séduit ou, à l'inverse, irrité par ce qu'on lui propose. Mais le mérite indiscutable de la pièce consiste à provoquer un questionnement de l'auditoire sur le son rapport au langage, et au monde. Pour Ariane Martinez, la réticence au théâtre s'inscrit également dans les gestes avortés. Aussi s'intéresse-t-elle à la résurrection de la pantomime dans les années 1880. L'écriture de ce genre particulier se construit paradoxalement, en énonçant la parole dans le texte, et en la taisant lors de la représentation. Les formes muettes du théâtre interpellent les spectateurs qui, face à des interlocuteurs réticents, ressentent souvent un gêne, une impression de manque ou d'inachevé. Ariane Martinez reste d'ailleurs sans complaisance avec son sujet : « la pantomime fin-de-siècle éclaire ainsi le caractère éminemment paradoxal de la réticence, et son impossibilité à être érigée en principe stable» (238), nous dit-elle. A chaque article son époque de prédilection, et c'est à la comédie italienne de la Renaissance que s'intéresse Bianca Concolino Mancini Abram. Elle analyse une comédie de Girolamo Bargagli, préfigurant la tragi-comédie, et portant sur le thème de la vérité déguisée : La Pellegrina (1567). Les spectateurs doivent, à l'occasion, inférer du sens à partir des propos tenus par les personnages, propos rendus encore plus opaques par les déguisements qu'ils portent. L'auteur fait une analyse serrée du texte dramatique, mettant l'accent sur la construction du discours, avant de conclure par l'épilogue de la pièce, où le dévoilement littéral du personnage en vient à signifier "l'abandon de toute réticence » (248). Avec Giovanni Iaquinta, nous abordons le septième art par le biais du cinéma de Nanni Moretti. C'est au dispositif de représentation réticente élaboré par l'Italien que s'intéresse l'auteur, à l'affût des propos susceptibles de créer chez le spectateur une incertitude interprétative. Interruptions visuelles et hors champ finissent par contaminer le récit, qui devient lui-même réticent. L'article montre ensuite comment le pacte autobiographique instauré par le réalisateur va à l'encontre des attentes du spectateur, juxtaposant des éléments authentiques et des séquences de fiction. La réticence, pour Moretti, serait par conséquent une attitude, une façon d'être proche de l'ironie, qui exprimerait l'impossibilité de trouver la vérité absolue grâce au savoir. 
Quant à la réticence étudiée par Katalin Kovacs, elle est d'ordre discursif : dans les Salons de Diderot - mué en critique d'art commentant les tableaux de Boucher ou de Chardin - l'auteur se penche sur «la résistance de certains types d'image face à la parole du critique qui essaie de les commenter » (265). On apprend que pour Diderot, la réticence, proche de l'aposiopèse, semble tantôt révéler l'échec de la parole face à une œuvre prolixe, tantôt trahir une admiration telle que les mots n'ont plus lieu d'être.

6 La cinquième et dernière partie de l'ouvrage, "Proses Réticentes ", est aussi la plus longue. Elle s'ouvre sur un article d'Anne Coudreuse, qui observe de plus près deux romans de Rétif de la Bretonne. Etudiant les dispositifs textuels de la réticence, elle passe en revue les noms tronqués, les périphrases, les allusions, les indications de suppression, les italiques ou les points de suspension. En fin de compte, elle démontre avec succès le caractère particulier de la réticence dans l'esthétique des Lumières. L'esthétique balzacienne est très différente; mais comme le montre Nathalie Solomon, les réticences sont elles aussi récurrentes chez Balzac, provoquant tout à la fois le désir et l'exaspération du lecteur. Fausses pistes, ou indices parsemés dans le récit, créent un déficit stimulant. Pareille réserve donne au récit une force singulière, puisque derrière ce qui est raconté, se profilent d'autres récits à ré-inventer lors de chaque nouvelle relecture. Sans transition, Richard Pedot fait une contribution sur les interprétations de Bartleby, figure de la réticence créée par Melville. L'auteur ne revient pas tant sur le texte en lui-même que sur quelques grands commentateurs, et s'attarde sur Deleuze, attentif aux «replis» du texte. Sont donc analysés les rapports entre philosophie et critique, à partir du silence de Bartleby. On voit alors comment la littérature répondrait à une nécessité absolue, inexplicable, consistant à «transformer l'absence de récit en récit de l'absence " (321). Josiane Paccaud-Huguet choisit quant à elle le roman moderne afin d'y mettre en lumière les « rets du sens ", pour reprendre la jolie formule de son titre. Elle livre une analyse très fine de The Aspern Papers, poème où la lettre poétique se dérobe à la lecture, ou encore récit clos par une ultime aposiopèse. Vient ensuite le célèbre Heart of Darkness de Joseph Conrad, texte remarquable pour son «traitement artistique du lieu du silence» (333), dont l'auteur admire la retenue et souligne qu'il est en fin de compte poiésis : il montre l'indicible. Puis, avec « Rituels de l'inéchangé, A Pale View of Hills de Kazuo Ishiguro ", Chantal Delourme s'attache à la particularité d'un roman qui fait co-exister la réticence avec la violence. Le texte d'Ishiguro met en scène des rituels d'échange où la parole s'estompe, où le discours porte les traces de ce qu'on lui a ôté, où parler relève de l'impossible. L'auteur met également en lumière l'écriture de soi, empreinte d'affleurements et d'esquives, de ruptures et de glissements. Enfin est abordée la notion de contretemps à la lumière des écrits de Paul Ricoeur, menant à la conclusion selon laquelle «la réticence interprétative serait (...) la condition d'un questionnement éthique " (355). La contribution de Stéphanie Horace concerne encore un autre domaine: le nouveau roman. Pour Simon Duras ou Robbe-Grillet, constate-t-elle, la réticence va de pair avec la répétition, voire même le ressassement. Les points de suspension, par exemple, marquent fréquemment une interruption dans le nouveau roman, fondé sur un texte lacunaire. Le lecteur doit alors mener sa propre enquête pour débusquer le sens, car la réticence radicalise l'énigme. De plus, une écriture résistant à la lecture se double de récurrences, car le mensonge, lui, se répand partout. Le nouveau roman relèverait donc à la fois du manque et de la prolifération. L'article suivant retient immédiatement l'attention, dans la mesure où se pose un sujet rarement traité dans ce genre d'ouvrage: Catherine Tauveron fait une place d'honneur à la littérature de jeunesse. 
Elle reprend elle aussi la dialectique réticence/prolifération et tâche de nous faire comprendre comment de la réticence, l'enfant va extirper du sens. Son intellect est stimulé par le non-dit (parfois forme de pudeur), et selon qu'il rencontre des «blancs récupérables et monosémiques" (380) ou «des blancs non recouvrables et polysémiques " (381), il va tisser du sens de manière différente. Les récits adaptés aux jeunes lecteurs misent sur le retard d'informations, sur le camouflage du centre de la perception, procèdent par allusions, jouent sur la mauvaise foi des personnages. L'auteur redonne donc à cette littérature complexe des lettres de noblesse qu'elle n'aurait jamais dû perdre, insistant sur le fait quelle est « un merveilleux tremplin pour accéder un jour à la littérature tout court » (387). La dernière contribution venant clore ce très riche ouvrage est celle de Catherine Viollet, qui se penche sur le cas des jeunes diaristes russes du dix-neuvième siècle. Ils expriment parfois une certaine réticence à se livrer, et restent sur la réserve, car écrire l'intimité reste toujours délicat. En outre, rédiger un journal au dix-neuvième siècle était pour les jeunes gens un exercice imposé par la famille: dès lors, on comprend mieux la réticence des diaristes, soumis au contrôle de leurs proches. Rien d'étonnant, en fin de compte, à ce que les réticences exprimées dans ces journaux frisent l'autocensure.

7 Au total, ce numéro de la Licorne, grâce à des optiques variées sur un sujet ambivalent, est une réussite. Ecrire sur le non-dit était pourtant une gageure. Comment s'étendre sur une réserve? Comment définir une notion fuyante, qui se dérobe à toute explication définitive? Le pari était osé, et l'on aurait pu craindre une certaine redondance, ou une certaine opacité dans les propos tenus. Mais les études proposées sont foisonnantes, stimulantes, et ne recourent jamais aux simplifications abusives. La démarche scientifique de chaque intervenant est clairement exposée, et les bibliographies proposées, très diverses, pourront servir d'outils de travail dans tous les domaines: linguistiques, philosophiques, littéraires, ou visuels. Chacun, selon sa spécialité, pourra donc y trouver son compte...

INDEX

Thèmes : Recensions

\section{AUTEUR}

\section{SOPHIE CHIARI-LASSERRE}

Université de Provence 